\title{
SOC estimation based on LPV-Kalman considering coulometric conversion
}

\author{
Weiming Sun ${ }^{1, \mathrm{a}}$, Yanping Zheng ${ }^{1, \mathrm{~b}}$, Xincan Wang ${ }^{1, \mathrm{c}}$ \\ NanJing Forest University, NanJing, JiangSu, 210037, China \\ a529073072@qq.com, b2402782773@qq.com, c821028656@qq.com
}

Keywords: Lithium-ion battery, SOC(state of charge) estimation, coulometric conversion coefficient, LPV(linear parameter-varying) model.

Abstract: $\mathrm{C} / 3$ discharging rate has been used to define battery nominal capacity in Chinese national standard. According to definition of the battery state of charge by the United States Advanced Battery Consortium, this article proposes that the capacity in different charging or discharging current should be unified to $\mathrm{C} / 3$ discharging capacity by the coulometric conversion coefficient. Through parameter identification of battery equivalent circuit model, this paper embeds model parameters to LPV model considering the coulometric conversion coefficient and uses LPV-Kalman to estimate SOC in time. Then, it compares to SOC estimation considering traditional Coulomb efficiency. Experiment shows that, this SOC estimation method has higher accuracy.

\section{Introduction}

As environmental and energy issues have become increasingly prominent, lithium-ion is more suitable for electric vehicles because of non-polluting, high energy density and long life cycle[1]. Realtime and accurate SOC estimation is one of the key technologies to apply lithium-ion battery to electric vehicles. The most commonly methods which use to estimate the battery SOC are Ah, the open circuit voltage, neural networks and Kalman filtering method[2]. However, when used to test the actual driving conditions in the car, these algorithms are insufficient in accuracy and timeliness .

LPV system is a special kind of time-varying systems. Its model coefficient matrix is a function about external measurable parameter. The change can reflect the nonlinear characteristics of the system and does not require linear process[3]. LPV has a great engineering and theoretical significance. In the car driving conditions, the battery system is nonlinear time-varying systems. It can be changed to LVP system through the transformation.

State of charge is a ratio between remaining battery capacity and the nominal battery capacity at a specified discharging rate by United States Advanced Battery Consortium (USABC). The battery rated capacity is different with different charge-discharge rate. Battery's rated capacity is defined by predetermined condition with $\mathrm{C} / 3$ discharging rate in GB/T18332.2-2001 in china. Therefore, this article considered that charging and discharging capacity in different current should be unified to $\mathrm{C} / 3$ discharging capacity through the coulometric conversion coefficient when calculating the battery SOC. Thus, based on lithium-ion battery that capacity is $10 \mathrm{Ah}$ and voltage is $3.2 \mathrm{~V}$, this paper proposes a realtime battery SOC estimation based on LPV model considering coulometric conversion coefficient. Through parameter identification of battery equivalent circuit model, model parameter identification has been embedded to LPV model considering the coulometric conversion coefficient to carry out joint simulation. Then compare the difference between the result considering the coulometric and the result considering coulomb efficiency. 


\section{coulometric conversion coefficient curve}

There are different values between charging capacity and discharging capacity because of the impacts of the battery internal resistance, charge and discharging rate , temperature, cycle life and so on. In the SOC estimation, battery changes in capacity should be considered caused by these factors. Coulomb efficiency is often adopted to describe it. Coulomb efficiency $\eta$ of the current SOC estimation is the ratio between capacity $\left(\mathrm{Q}_{\mathrm{ID}}\right)$ with different discharging rate and capacity $\left(\mathrm{Q}_{\mathrm{n}}\right)$ with standard discharging rate, which is $\eta=\mathrm{Q}_{\mathrm{ID}} / \mathrm{Q}_{\mathrm{n}}$. However, there are the discharging and charging processes in the same time in the driving conditions. Coulomb efficiency should be described by the charging and discharging processes. So in this paper, the coulometric conversion coefficient has been proposed.

Specific steps for the coulometric conversion coefficient as follows. (a) The battery of discharging termination voltage under standard conditions with $\mathrm{C} / 3$ rate constant current is charged to the charging termination voltage. (b) The nominal charging capacity $\mathrm{Q}_{\mathrm{SC}}$ is calculated by Ah method. (c) The battery of charging termination voltage under standard conditions with $\mathrm{C} / 3$ rate constant current is discharged to the discharging termination voltage. (d) The nominal charging capacity $\mathrm{Q}_{\mathrm{SD}}$ is calculated by Ah method. (e) Charging capacity value $\mathrm{Q}_{\text {IC }}$ and discharging capacity value $\mathrm{Q}_{\mathrm{ID}}$ are calculated by the same method with different constant charging or discharging current $\mathrm{I}$. (f) Then charging coulometric conversion coefficient $\mathrm{K}_{\mathrm{IC}}=\left(\mathrm{Q}_{\mathrm{SD}} / \mathrm{Q}_{\mathrm{SC}}\right) *\left(\mathrm{Q}_{\mathrm{SC}} / \mathrm{Q}_{\mathrm{IC}}\right)=\mathrm{Q}_{\mathrm{SD}} / \mathrm{Q}_{\mathrm{IC}}$ and discharging coulometric conversion coefficient $\mathrm{K}_{\mathrm{ID}}=\mathrm{Q}_{\mathrm{SD}} / \mathrm{Q}_{\mathrm{ID}}$ with different constant charging and discharging current $\mathrm{I}$ is calculated by formula. During the test period, assuming that the lithium-ion battery voltage has recovered to a stable average value after it maintains still in $1 \mathrm{~h}[4]$.

The charging coulometric conversion coefficient $\mathrm{K}_{\mathrm{IC}}$, discharging coulometric conversion coefficient $\mathrm{K}_{\mathrm{ID}}$ have been fitted, as they are shown in Fig.2 and 3. And the relationships between coulometric conversion coefficient and the current I are shown as follows.

$\mathrm{K}_{\mathrm{IC}}=-0.001 \mathrm{I}^{4}+0.041 \mathrm{I}^{3}-0.397 \mathrm{I}^{2}+1.606 \mathrm{I}+95.732$

$\mathrm{K}_{\mathrm{ID}}=-0.0026 \mathrm{I}^{4}+0.096 \mathrm{I}^{3}-0.941 \mathrm{I}^{2}+3.569 \mathrm{I}+95.494$

Converting capacity with other conditions into capacity with $\mathrm{C} / 3$ charge-discharging rate, then calculate the battery SOC as follow.

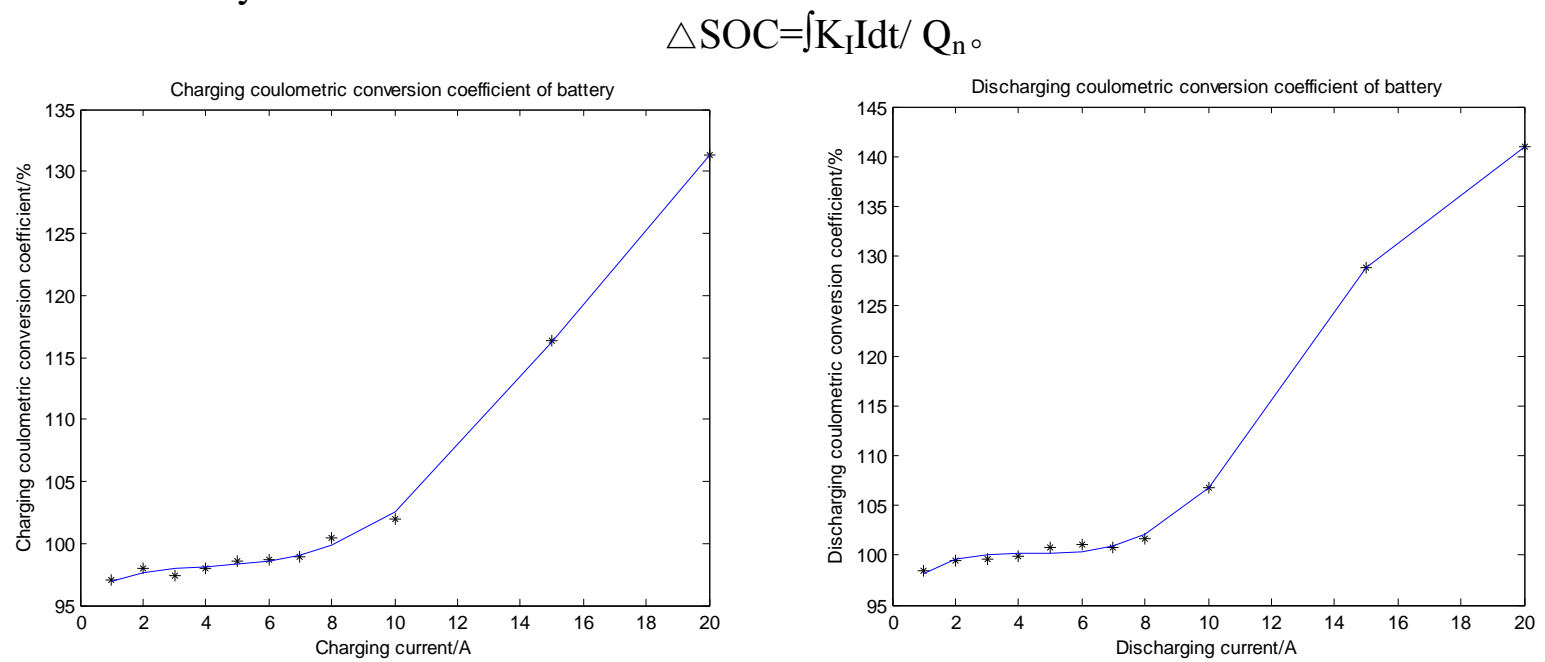

Fig.1 Charging coulometric conversion coefficient of battery (left)

Fig.2 Discharging coulometric conversion coefficient of battery (right) 


\section{Lithium-ion equivalent circuit model}

\section{Equivalent circuit model}

In the commonly used battery equivalent circuit model, Rint model is too simple, complexity of GNL model is too high, the advantages of PNGV model is limited to a dynamic environment. Considering the complexity and practicality of the equivalent circuit model, The charging and discharging characteristics of the battery is described by Second equivalent circuit model shown in Figure 3[5].

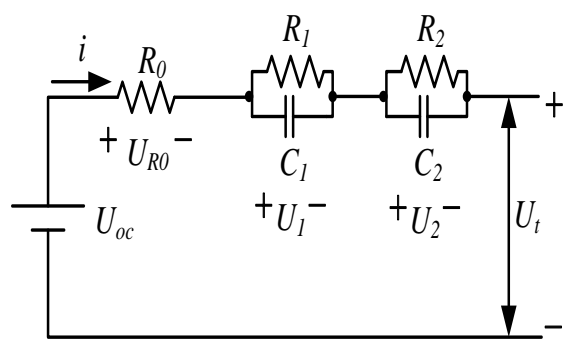

Fig.3 second-order RC circuit model

Wherein, $\mathrm{U}_{\mathrm{t}}$ indicates the battery terminal voltage. $\mathrm{R}_{0}$ represents ohmic resistance. $\mathrm{R}_{1}$ is electrochemical polarization resistance. $C_{1}$ is electrochemical polarization capacitance. $R_{2}$ is a concentration polarization resistance. $\mathrm{C}_{2}$ is concentration polarization capacitance. Two parallel links which contain $\mathrm{R}_{1}, \mathrm{C}_{1}, \mathrm{R}_{2}$, and $\mathrm{C}_{2}$ simulate battery electrochemical polarization and concentration polarization effect. Uoc represents the open circuit voltage.

\section{Parameter identification of battery equivalent circuit model}

Model parameters include the ohmic resistance $R_{0}$, electrochemical polarization resistance $R_{1}$ and the capacitance $\mathrm{C}_{1}$, the concentration polarization resistance $\mathrm{R}_{2}$ and the capacitance $\mathrm{C}_{2}$. Based on hybrid pulse power characterization, performance tests have been carried out. Test programs are as follows. Battery is discharged by 5A pulse discharging current which maintains $48 \mathrm{~S}$. Battery is still in $10 \mathrm{~min}$ after discharged. This process can be divided into instantaneous voltage drop process when the current is loaded, zero-input response process and zero state response.

Instantaneous voltage drop is mainly caused by the ohmic resistance $\mathrm{R}_{0}$, according to Ohm's law, $\mathrm{R}_{0}$ is calculated as follow.

$R_{0}=\Delta U / I$

(1)

Within 10min after the end of the current load, capacitances $C_{1}$ and $C_{2}$ discharge through their respective resistances $\mathrm{R}_{1}$ and $\mathrm{R}_{2}$ of $\mathrm{RC}$ circuit. this is zero-input response. the response is expressed as follow.

$$
U_{c}=U_{o c v}-U_{t}=U_{1} e^{-t / \tau_{1}}+U_{2} e^{-t / \tau_{2}}
$$

Where, $\mathrm{U}_{\mathrm{c}}$ is the sum of voltage on capacitance $\mathrm{C}_{1}$ and $\mathrm{C}_{2} . \mathrm{U}_{1}$ and $\mathrm{U}_{2}$ are initial voltage on capacitances $\mathrm{C}_{1}$ and $\mathrm{C}_{2} . \mathrm{U}_{\mathrm{t}}$ is the terminal voltage of the battery. $T_{1}, T_{2}$ are discharging time constant. The $\mathrm{U}_{1}, \mathrm{U}_{2}, \mathrm{~T}_{1}, \mathrm{~T}_{2}$ is undetermined coefficients. They are identified by least squares and $\mathrm{T}_{1}$, $\mathrm{T}_{2}$ is determined.

The process loading current which maintains $48 \mathrm{~s}$ is the zero state response. The process is expressed as follow.

$U_{c}=U_{k}-U_{t}=I R_{1}\left(1-e^{-t / \tau_{1}}\right)+I R_{2}\left(1-e^{-t / \tau_{2}}\right)$ 
Where: $\mathrm{U}_{\mathrm{k}}$ is the battery terminal voltage after loading the current moment. $\mathrm{T}_{1}, \mathrm{~T}_{2}$ are substituted into the equation (3). $R_{1}, R_{2}$ is undetermined coefficients. $R_{1}, R_{2}$ can be obtained by least squares. Then according to ${ }_{1}=\mathrm{R}_{1} * \mathrm{C}_{1}, \mathrm{~T} \quad 2=\mathrm{R}_{2} * \mathrm{C}_{2}, \mathrm{C}_{1}$ and $\mathrm{C}_{2}$ can be obtained.

Tab.1 Results of model parameter identification of resistance and capacitance

\begin{tabular}{cccccc}
\hline SOC & $R_{0} / \mathrm{m} \Omega$ & $R_{1} / \mathrm{m} \Omega$ & $R_{2} / \mathrm{m} \Omega$ & $C_{1} / \mathrm{F}$ & $C_{2} / \mathrm{F}$ \\
\hline 0.1 & 41.02 & 41.12 & 29.64 & 6.09 & 1613.3 \\
0.2 & 38.04 & 38.48 & 13.48 & 6.31 & 1631.26 \\
0.3 & 39.86 & 40.18 & 11.97 & 6.33 & 2312.36 \\
0.4 & 38.62 & 39.04 & 10.76 & 6.71 & 2452.52 \\
0.5 & 36.64 & 37.06 & 9.98 & 7.1 & 2531.94 \\
0.6 & 35.4 & 34.96 & 9.52 & 7.8 & 1932.93 \\
0.7 & 33.28 & 33.96 & 10.86 & 8.65 & 2406.42 \\
0.8 & 32.88 & 33.16 & 10.2 & 7.65 & 2747.39 \\
0.9 & 30.84 & 31.58 & 9.01 & 9.46 & 2861.63 \\
1 & 31.72 & 31.84 & 30.02 & 11.28 & 791.11 \\
\hline
\end{tabular}

\section{LPV model considering coulometric conversion coefficient}

Although Extended Kalman Filter can solve nonlinear system problem, it requires a first-order differentiable nonlinear systems and should calculate Jacobi matrix. And EKF is difficult to achieve high accuracy when dealing with strongly nonlinear systems. Taking the estimated accuracy of the algorithm and the computational complexity into account, LPV-Kalman filter algorithm is applied to estimate SOC[6]. According LPV theory, lithium-ion battery state space expression is shown as follow.

$$
\begin{aligned}
& {\left[\begin{array}{l}
z(k+1) \\
U_{1}(k+1) \\
U_{2}(k+1)
\end{array}\right]=\left[\begin{array}{ll}
1 & 0 \\
0 & A(\rho(t))
\end{array}\right]\left[\begin{array}{l}
z(k) \\
U_{1}(k) \\
U_{2}(k)
\end{array}\right]+\left[\begin{array}{c}
-\frac{K_{I} T_{s}}{3600 Q_{n}} \\
B(\rho(t))
\end{array}\right] \bullet i(k)+w(k)} \\
& U_{t}=C(\rho(t)) \cdot\left[\begin{array}{l}
z(k) \\
U_{1}(k) \\
U_{2}(k)
\end{array}\right]+D(\rho(t)) \cdot i(k)+v(k)
\end{aligned}
$$

Where $\mathrm{w}(\mathrm{k})$ and $\mathrm{v}(\mathrm{k})$ respectively represent the system noise and measurement noise, and they are independent. $T_{S}$ is system sampling time. $K_{I}$ is coulometric conversion coefficient. $A(\rho(t)), B(\rho(t))$, C $(\rho(t)), D(\rho(t))$ are function of scheduling variables. The expressions of them are shown as follows.

$$
A(\rho(t))=\left[\begin{array}{cc}
\exp \left(-\frac{T_{s}}{R_{1}(z(t)) \cdot C_{1}(z(t))}\right) & 0 \\
0 & \exp \left(-\frac{T_{s}}{R_{2}(z(t)) \cdot C_{2}(z(t))}\right)
\end{array}\right]
$$


$B(\rho(t))=\left[\begin{array}{l}R_{1}(z(t)) \cdot\left[1-\exp \left(-\frac{T_{s}}{R_{1}(z(t)) \cdot C_{1}(z(t))}\right)\right] \\ R_{2}(z(t)) \cdot\left[1-\exp \left(-\frac{T_{s}}{R_{2}(z(t)) \cdot C_{2}(z(t))}\right)\right]\end{array}\right]$

(6)

$C(\rho(t))=\left[\begin{array}{lll}\frac{\partial \operatorname{UoCv}(z)}{\partial z} & -1 & -1\end{array}\right]$

(7)

$D(\rho(t))=-R_{0}$

$(8)$

\section{The simulation model to estimate SOC of the battery}

SOC estimation method with Kalman filter designed in this paper has a similar process with EKF, except that the system matrix is a function of scheduling variables. Flow chart of LPV-Kalman filter is shown in Figure 4.

LPV-Kalman filter simulation model designed in MATLAB / Simulink is shown in Figure 5. The left frame in Fig.5 is updating module of the package coefficient matrix, which inside the small box is the battery charging and discharging conversion coefficient module. the right frame is measurement module and updating module which is similar to Kalman filter method.

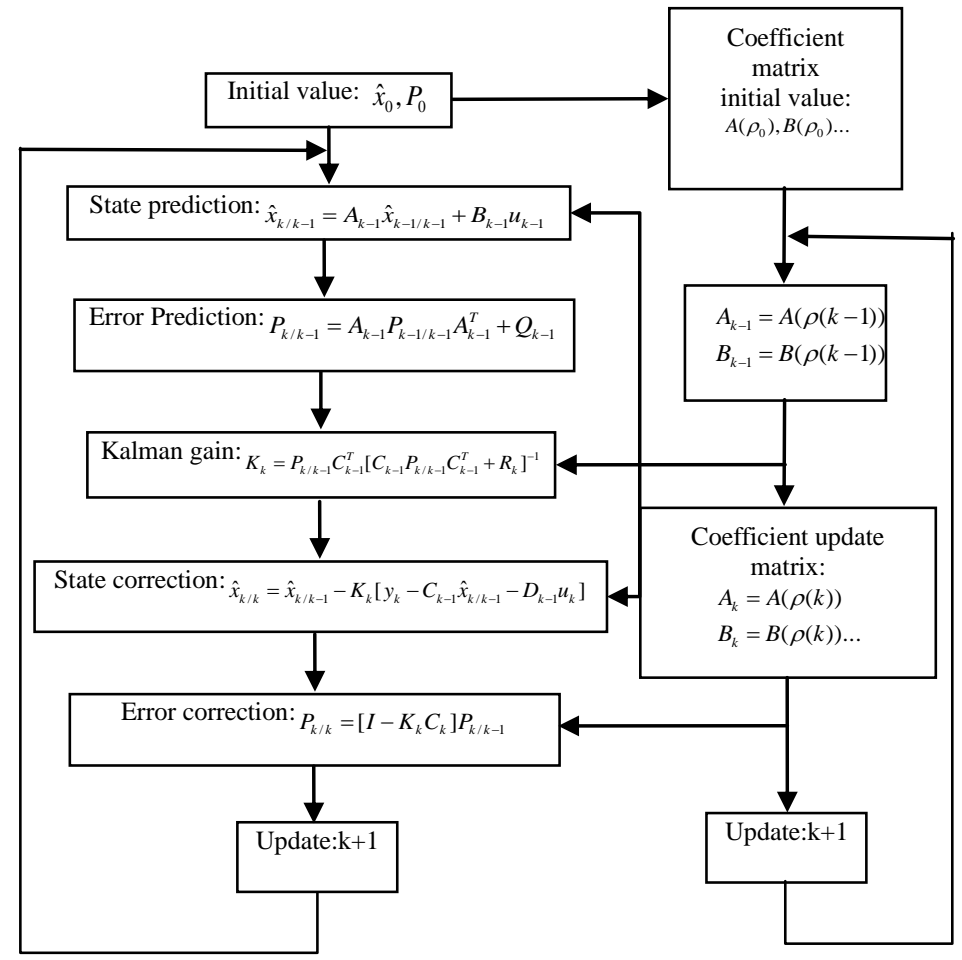

Fig.4 Flow chart of LPV-Kalman filter 


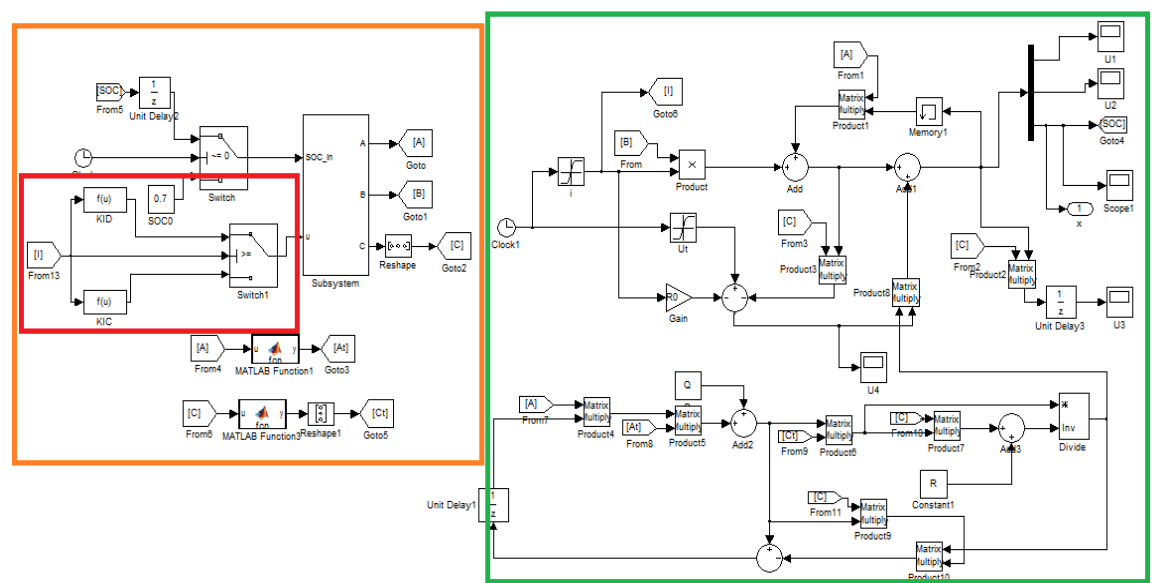

Fig.5 Simulation model of SOC estimation

To verify the effectiveness of LPV-Kalman filter algorithm and the difference between considering the coulometric conversion coefficient and considering coulomb efficiency, variable charging current condition test is designed. specific test is shown as follow. At first, the battery is charged with 10A that maintains 50s. battery is still in $10 \mathrm{~s}$ after charged. Then the battery is charged with $5 \mathrm{~A}$ current which maintains 120s. Battery is still in 20s after discharged. Finally, the battery is charged with $3 \mathrm{~A}$ that maintains 180s. Comparison between SOC simulation results and the experimental result is shown in Figure 6. Estimated value 1 is SOC estimated value considering the coulometric conversion coefficient, estimated value 2 is the estimated value considering traditional Coulomb efficiency. Simulation results show that LPV-Kalman filter algorithm has validity. And the error between estimated value1 and estimated value 2 becomes bigger with the increase of the charging conditions. It is shown in Figure 7.
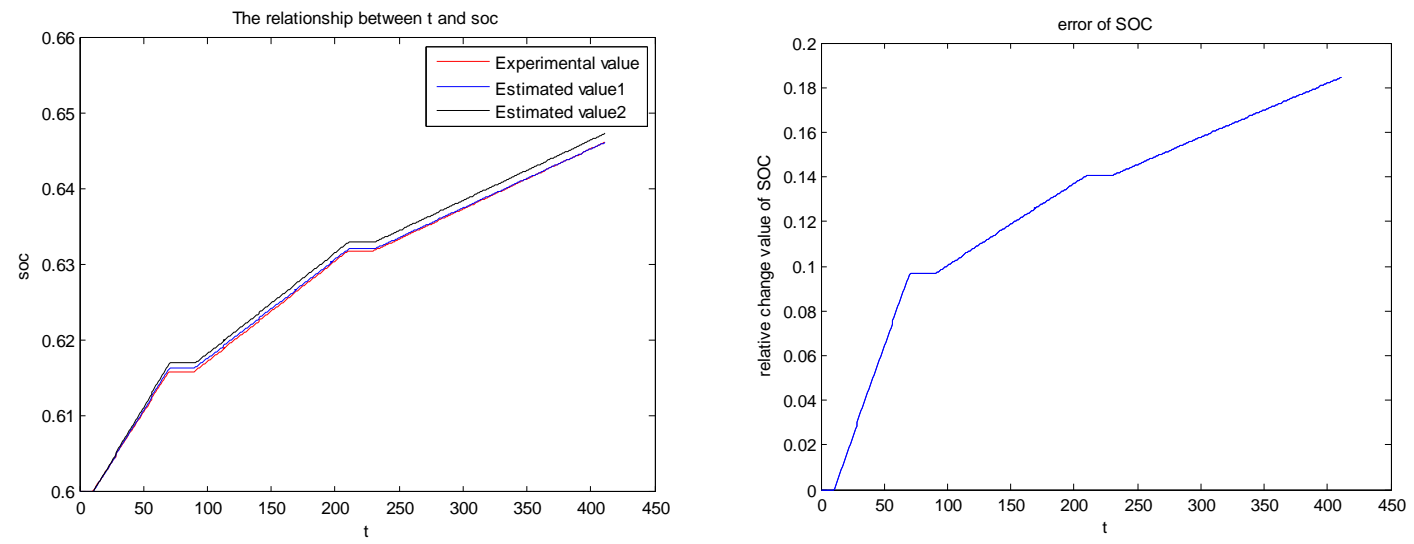

Fig.6 Comparison between simulation value and test value of SOC (left)

Fig.7 Error between simulation value1 and value2 of SOC (right)

\section{Conclusions}

In this paper, The following two things are completed. (a) through the coulometric conversion coefficient test, the fitted curves have been obtained, and apply them to SOC estimation. (b) Through parameter identification of battery equivalent circuit model, model parameter identification has been embedded to LPV model considering the coulometric conversion coefficient to carry out joint simulation. Then compare the difference between the result considering the coulometric and the result considering coulomb efficiency. 


\section{References}

[1] Yao He, XingTao Liu, ChenBin Zhang. A new model for State-of-charge (SOC) estimation for high-power Li-ion batteries[J].Applied Energy, vol.101, no.4, pp. 808-814, 2013.

[2] Gregory L. Plett. Extended Kalman filtering for battery management systems of LiPB-based HEV battery packs Part 3. State and parameter estimation[J]. Journal of Power Sources,vol. 134, no. 2, pp. 277-292, Aug. 2004.

[3] Saeed Sepasi, Reza Ghorbani, Bor Yann Liaw. A novel on-board state-of-charge estimation method for aged Li-ion batteries based on model adaptive extended Kalman filter[J]. Journal of Power Sources, vol. 245, no. 2, pp.337-344, 2014.

[4] Y. Hu, S. Yurkovich. Battery cell state-of-charge estimation using linear parameter varying system techniques[J]. Journal of Power Sources, vol.198, no.4, pp.338-350, 2012.

[5] D.Riu, M.Montaru, Y.Bultel. Time domain simulation of Li-ion batteries using non-integer order equivalent electrical circuit[J]. Commum Nonlinear Sci Numer Simulat, vol.18, pp,1454-1462, 2013.

[6] Zheng Yanping, Du Zhengping, WU Songsong. A real-time SOC estimation method of LiFePO4 Li-ion battery based on LPV model[J]. JOURNAL OF HEFEI UNIVERSITY OF TECHNOLOGY, vol. 37, no. 5, pp. 600-603, 2014.(in Chinese) 